\title{
$\beta$ endorphin concentrations in PBMC of patients with different clinical phenotypes of multiple sclerosis
}

\author{
M Gironi, R Furlan, M Rovaris, G Comi, M Filippi, A E Panerai, P Sacerdote
}

J Neurol Neurosurg Psychiatry 2003;74:495-497

The possible link between the opioid peptide $\beta$ endorphin and the heterogeneity of the clinical course of multiple sclerosis (MS) was investigated. Peripheral blood mononuclear cells (PBMC) concentrations of $\beta$ endorphin were measured in 50 patients in different phases of MS. Thirty nine patients also underwent post-contrast magnetic resonance imaging of the brain. Among MS forms, the highest $\beta$ endorphin concentrations were found in PBMC from patients with relapsing remitting MS and the lowest in patients with the progressive forms. Average $\beta$ endorphin concentrations were lower, although not significantly, in patients with than in those without magnetic resonance imaging enhanced lesions. These data suggest that $\beta$ endorphin may have a role in the downregulation of the inflammatory process.

$\mathrm{T}$ he immunological mechanisms of multiple sclerosis (MS) are only partially defined, but they are known to differ between patients and in different phases of the disease. ${ }^{1}$ Several studies have shown a pronounced increase of Thl cytokines in patients with active disease, ${ }^{2}$ whereas interleukin 10 , transforming growth factor $\beta$, and interleukin 4 have been found to be downregulated during phases of disease activity and upregulated during phases of disease remission. ${ }^{3}$ Among non-conventional immune mediators, the opioid peptide $\beta$ endorphin has been shown to affect some immune responses, which in turn modulate cytokine production. ${ }^{4} \beta$ Endorphin is synthesised by cells of the central nervous system (arcuate nucleus) and immune system (lymphocytes, thymocytes, monocytes, and splenocytes). ${ }^{5}$ We have previously evaluated $\beta$ endorphin concentrations in peripheral blood mononuclear cells (PBMC) of patients with MS. We found reduced concentrations of PBMC $\beta$ endorphin in patients compared with healthy controls, increased concentrations of $\beta$ endorphin during interferon beta treatment compared with basal values, and increased concentrations of $\beta$ endorphin soon after a clinical relapse. ${ }^{4}$ In this study, we investigated the possible role of $\beta$ endorphin in determining the heterogeneity of the course of MS. To this end, we measured $\beta$ endorphin concentrations in PBMC from patients with MS with clinically different phenotypes or in different phases of the disease.

\section{PATIENTS AND METHODS}

Fifty patients with clinically definite $\mathrm{MS}^{6}$ and 12 healthy controls entered the study. We classified MS into relapsing remitting, primary progressive, secondary progressive, benign, and clinically relapsing, according to established clinical criteria ${ }^{6}$ (table 1).

None of the patients had ever taken disease modifying treatments (such as interferons, glatiramer acetate, mithoxantrone) or were being treated with neuroleptic, antidepressant, or antiepileptic drugs.

Patients experiencing relapses started steroid treatment after blood sample collection

$\beta$ Endorphin concentrations were also measured in 12 healthy controls (five men and seven women) with a mean (SD) age of 40.0 (4.4) years.

In all patients and controls, peripheral blood samples were collected in a tube containing EDTA, and PBMC were separated by gradient sedimentation over Ficoll-Paque (Pharmacia, Uppsala, Sweden). Aprotinin (Boehringer Ingelheim Pharmaceutical Inc, Ridgefield, Connecticut, USA) 1000 kIU was added to all samples before storage at $-20^{\circ}$ to inhibit peptide degradation. Cells were resuspended in $1 \mathrm{ml}$ of 0.1 normal acetic acid, homogenised, and centrifuged. Supernatants were frozen for radioimmunoassay. ${ }^{4}$ The antiserum and radiommunoassay procedures to measure $\beta$ endorphin were previously described and validated. ${ }^{4}$ The antiserum used was directed to the $C$ terminal sequence of human $\beta$ endorphin.

Sensitivity of the method was $10 \mathrm{pg}$ per tube, and intra-assay and interassay variation coefficients were $8 \%$ and $11 \%$ respectively.

In 39 patients, dual echo spin-echo and post-contrast (intravenous gadolinium-DTPA, $0.1 \mathrm{mmol} / \mathrm{kg}$ ) Tl weighted spin-echo magnetic resonance images of the brain were also obtained within 48 hours after blood sample collection. Images were acquired with a $1.5 \mathrm{~T}$ scanner, with full brain coverage (the scan geometry was 24 contiguous, axial, $5 \mathrm{~mm}$ thick slices with approximately $1 \times 1 \mathrm{~mm}$ in-plane pixel resolution). The presence and number of gadolinium enhanced

Abbreviations: MS, multiple sclerosis; PBMC, peripheral blood mononuclear cells

Table 1 Clinical and demographic characteristics of patients with multiple sclerosis

\begin{tabular}{llllll}
\hline Subgroup & \multicolumn{1}{l}{ Number of patients } & Male/female & Age (years) & EDSS score & Disease duration (years) \\
\hline Relapsing remitting & 17 & $6 / 11$ & $35.7(7.7)$ & $2.6(1.5)$ & $6.8(6.8)$ \\
Primary progressive & 10 & $5 / 5$ & $41.6(10.7)$ & $4.1(1.5)$ & $8.5(7.4)$ \\
Secondary progressive & 8 & $4 / 4$ & $42.6(7.1)$ & $4.1(1.2)$ & $10.6(6.8)$ \\
Benign & 5 & $0 / 5$ & $45.2(6.6)$ & $2.3(0.9)$ & $19.8(4.9)$ \\
Clinically relapsing & 10 & $3 / 7$ & $37.2(8.3)$ & $3.15(2.0)$ & $8.9(6.0)$ \\
Healthy controls & 12 & $5 / 7$ & $40.0(4.4)$ & & \\
\hline
\end{tabular}

Data are mean (SD). EDSS, expanded disability status scale. 

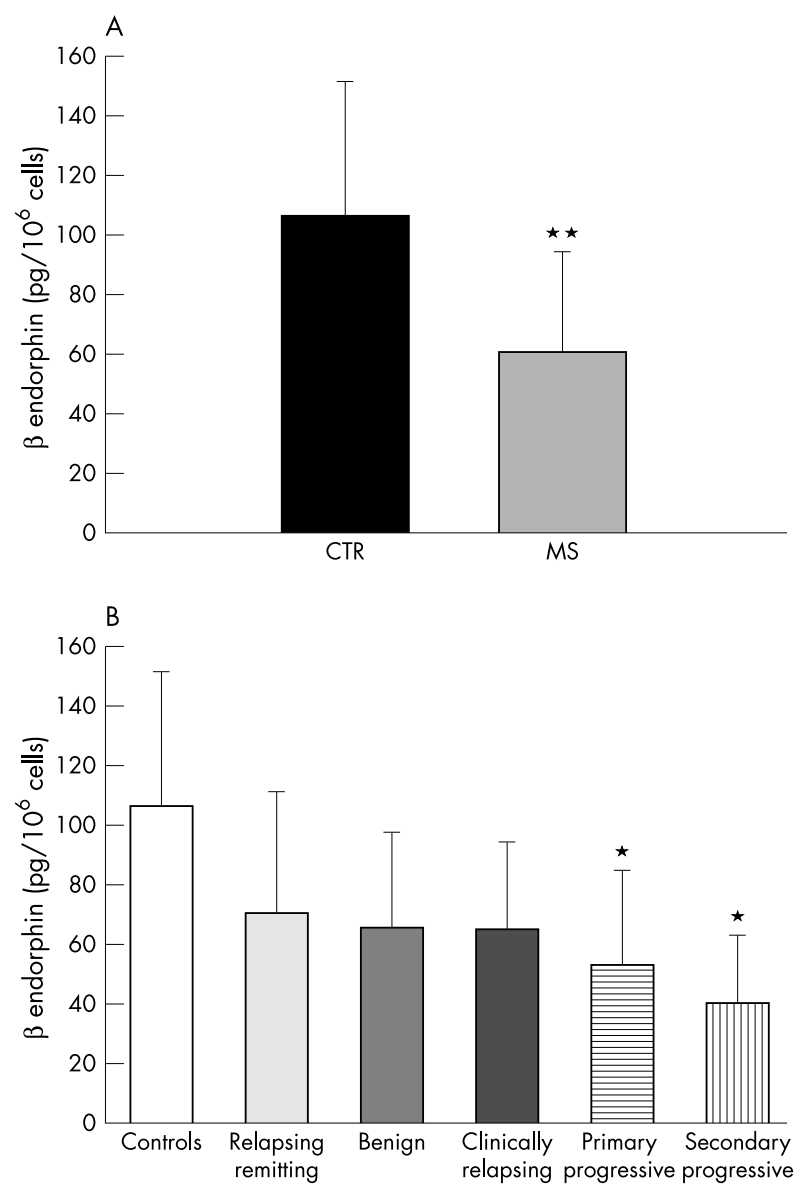

Figure 1 (A) $\beta$ Endorphin concentrations in peripheral blood mononuclear cells (PBMC) of patients with multiple sclerosis (MS) and of healthy control (CTR). (B) PBMC $\beta$ endorphin concentrations in various MS clinical phenotypes. Values are mean (SD). ${ }^{*} p<0.05$; ${ }^{* *} p<0.001$

lesions were assessed by an experienced observer following published guidelines. ${ }^{7}$

Differences in $\beta$ endorphin concentrations were analysed by analysis of variance, followed by post hoc Bonferroni corrected $t$ test. Correlations were evaluated with the Pearson product moment correlation.

\section{RESULTS}

$\beta$ Endorphin concentrations in PBMC samples obtained from all five groups of MS patients were significantly lower than those found in healthy controls (fig lA).

PBMC $\beta$ endorphin concentrations in the different disease groups are shown in fig $1 \mathrm{~B}$.

The highest $\beta$ endorphin concentrations were found in PBMC from patiens with relapsing remitting, benign, and clinically relapsing forms of MS.

Patients with primary and secondary progressive MS had the lowest $\beta$ endorphin concentrations that were significantly different from those of healthy controls.

Average $\beta$ endorphin concentrations were 50.2 (35.6) and $62.5(37.8) \mathrm{pg} / 10^{6}$ cells in patients with $(\mathrm{n}=11)$ and those without $(n=28)$ gadolinium enhanced lesions. This difference was not significant.

No significant correlation was found between the concentrations of $\beta$ endorphin and patients' age, disease duration, and severity (as measured by the expanded disability status scale score).

\section{DISCUSSION}

The results of this study confirm our previous data ${ }^{4}$ showing reduced $\beta$ endorphin concentrations in the immune cells of patients with MS compared with controls. This group of controls was very well matched with the patients in terms of age and sex, and their health condition was carefully checked to rule out any past or ongoing disease. Moreover, the concentrations of PBMC $\beta$ endorphin in the present group of controls was comparable with those found in other groups of controls. ${ }^{4}$

As is the case for other autoimmune diseases, MS is supposed to result from an imbalance between proinflammatory and anti-inflammatory factors. As in other autoimmune diseases (such as rheumatoid arthritis and Crohn's disease), $\beta$ endorphin concentrations are decreased in MS. ${ }^{4}$

The aim of this study was investigating a possible role of $\beta$ endorphin in the mechanisms leading to different clinical expressions of MS. So far, classifications of MS subtypes have been based largely on clinical phenomenology, but there is growing evidence that the subgroups of MS also differ with respect to epidemiology, pathogenesis, genetics, neuropathology, and neuroimaging.

In our study, the lowest PBMC $\beta$ endorphin concentrations were linked to the progressive forms of MS; that is, primary progressive and secondary progressive forms. On the other hand, patients with "benign" and relapsing remitting forms have the highest concentrations.

It remains to be elucidated whether the $\beta$ endorphin decrement should be considered a mere epiphenomenon of MS progression or rather to be a factor contributing to it.

However, data obtained in experimental autoimmune encephalomyelitis, an animal model of MS, showed a worsening of disease severity after $\beta$ endorphin blockade achieved with an opioid antagonist, ${ }^{8}$ suggesting a potentially protective role for $\beta$ endorphin in the pathogenetic mechanisms of MS.

In our previous study, we obtained other data leading to speculation that $\beta$ endorphin has a protective role. We found an increase of $\beta$ endorphin concentrations during interferon beta treatment. These results suggest that the opioid can be involved in the downregulation of the inflammatory process, present in MS.

A possible mechanism for this protective role of $\beta$ endorphin may be an involvement in cytokine balance. $\beta$ Endorphin is known to shift the Thl/Th2 balance towards Th2, ${ }^{49}$ while the removal of the opioid tone by the antagonist naloxone induces an increase of Thl cytokines such as interleukin 2 and interferon gamma and a decrease of interleukin $4 .{ }^{9}$

Moreover, recent findings from our group suggest that the effects of opioids on Th1/Th2 balance can be mediated by a decrease of the production of interleukin 12 by macrophages. ${ }^{10}$ This observation may be particularly intriguing since macrophages and macrophage cytokines are considered to be central factors involved in the onset and progression of MS. ${ }^{411}$

The observation that $\beta$ endorphin concentrations were, on average, lower in patients with magnetic resonance imaging evidence of ongoing MS activity also supports the concept that this compound may have a role in downregulating MS inflammatory processes.

Clearly, we cannot completely rule out that mechanisms leading to MS progression may influence $\beta$ endorphin concentrations. A longitudinal study evaluating the modification of the peptide over time would be helpful in elucidating this point. However, measuring this opioid may be valuable in defining new characteristics of the various clinical phenotypes of MS. 


\section{Authors' affiliations}

M Gironi, R Furlan, M Rovaris, G Comi, M Filippi, Department of Neuroscience, San Raffaele Hospital, Milan, Italy

A E Panerai, P Sacerdote, Department of Pharmacology, University of Milan, Milan, Italy

Correspondence to: Dr M Gironi, San Raffaele, DIMER, Via Olgettina 48, 20132, Milan, Italy; mgironi@dongnocchi.it

Received 27 August 2002

In revised form 20 December 2002

Accepted 2 January 2003

\section{REFERENCES}

1 Lucchinetti CF, Bruck W, Rodriguez M, et al. Distinct pattern of multiple sclerosis pathology indicates heterogeneity on pathogenesis. Brain Pathol 1996:6:259-74.

2 Navikas V, He B, Link J, et al. Augmented expression of tumour necrosis factor-alpha and lymphotoxin in mononuclear cells in multiple sclerosis and optic neuritis. Brain 1996;119:213-23.

3 Clerici $M$, Saresella $M$, Trabattoni $D$, et al. Single-cell analysis of cytokine production show different immune profile in multiple sclerosis patients with active or quiescent disease. J Neuroimmunol $2001 ; 121: 88-101$
4 Gironi M, Martinelli V, Brambilla E, et al. Beta-endorphin concentrations in peripheral blood mononuclear cells of patients with multiple sclerosis. Arch Neurol 2000;57:1178-81.

5 Blalock JE. A molecular basis for bidirectional communication between the immune and neuroendocrine systems. Physiol Rev 1989;69:1-32.

6 McDonald WI, Compston A, Edan G, et al. Recommended diagnostic criteria for multiple sclerosis: guidelines from the international panel on the diagnosis of multiple sclerosis. Ann Neurol 2001;50:121-7.

7 Rovaris M, Barkhof F, Bastianello S, et al. Multiple sclerosis: interobserver agreement in reporting active lesions on serial brain MRI using conventional spin echo, fast spin echo, fast fluid-attenuated inversion recovery and post-contrast T1-weighted images. J Neurol 1999:246:920-5.

8 Panerai A, Radulovic J, Monastra G, et al. Beta-endorphin concentrations in brain areas and peritoneal macrophages in rats susceptible and resistant to experimental allergic encephalomyelitis: a possible relationship between tumor necrosis factor alpha and opioids in the disease. J Neurol 1994;51:169-76.

9 Sacerdote P, Manfredi B, Gaspani L, et al. The opioid antagonist naloxone induces a shift from type 2 to type 1 cytokine pattern in $\mathrm{BALB} / \mathrm{cJ}$ mice. Blood 2000;95:2031-6.

10 Sacerdote $\mathbf{P}$, Limiroli E, Gaspani L, et al. Modulation of macrophage cytokine production by morphine and specific opioid agonists [abstract] Inflammation Res 2001;50(suppl 3):127.

11 Moser M, Murphy KM. Dendritic cell regulation of TH1-TH2 development. Nat Immunol 2000;1:199-205. 